\title{
Quantum corrections to the noncommutative kink
}

\author{
R. A. Konoplya \\ Department of Physics, Kyoto University, Kyoto 606-8501, Japan \\ and \\ D. V. Vassilevich* \\ Instituto de Física, Universidade de São Paulo, \\ Caixa Postal 66318 CEP 05315-970, São Paulo, S.P., Brazil
}

October 25, 2018

\begin{abstract}
We calculate quantum corrections to the mass of noncommutative $\phi^{4}$ kink in $(1+1)$ dimensions for intermediate and large values of the noncommutativity parameter $\theta$. All one-loop divergences are removed by a mass renormalization (which is different from the one required in the topologically trivial sector). For large $\theta$ quantum corrections to the mass grow linearly with $\theta$ signaling about possible break down of the perturbative expansion.
\end{abstract}

\section{Introduction}

The problem of how to compute quantum corrections to the mass of $(1+1)$ dimensional solitons was first addressed by Dashen, Hasslacher and Neveu [1] in 1974. This work was followed by a decade of activities related to quantum properties of solitons, see [2,3]. The subject was re-opened in 1997 in the context of supersymmetric solitons [4-7] (see also reviews [8,9]). About the same time many methods of calculations appeared, the ones based on the heat kernel expansion are most important for us [10-14].

Previous studies of solitons in noncommutative (NC) theories (cf. [15, 16]) avoided the problem of quantum corrections to their mass, except for the work

\footnotetext{
* On leave from V. A. Fock Institute of Physics, St. Petersburg University, Russia. E.mail: dmitry (at)dfn.if.usp.br
} 
[17], where quantum correction to an NC sine-Gordon soliton were calculated in a couple of leading orders of the $\theta$-expansion. This paper also reported certain difficulties in calculating the mass shift, which are probably not specific to the model considered, but rather common to all time-space NC theories.

The present paper is devoted to calculation of quantum corrections to the mass of $\mathrm{NC}$ kink in $(1+1)$ dimensions. We do not use the $\theta$-expansion. On the contrary, we concentrate on finite and large values of the noncommutativity parameter. As in [17] we define the mass shift (the vacuum energy $E$ ) as, roughly speaking, half the sum of eigenfrequencies of fluctuations about the soliton. Such a definitions is hard to justify rigorously since in time-space NC theories where there is no canonical definition of energy. Some arguments in favor of treating eigenfrequencies as one particle energies can be found in [18]. Anyway, $E$ is useful for the discussion of quantum properties of solitons and renormalization.

This paper is organized as follows. The next section contain some preliminary material on the $\mathrm{NC}$ kink and fluctuations about it mostly taken from [19]. In sec. 3 we study spectral density of the fluctuation and renormalization of the vacuum energy (which is done by adding a mass counterterm to the classical action). Section 4 is devoted to numerical calculations of the vacuum energy for several values of the $\mathrm{NC}$ parameter $\theta=2,3,5,7,10$ (in some natural units). The bound state frequencies are evaluated with the help of the WKB method, while the contribution of the continuous spectrum is calculated by using an approximating square well potential. We find that quantum corrections to the vacuum energy grow linearly with $\theta$.

\section{Classical solution and fluctuations}

We take the classical action of $\mathrm{NC} \phi^{4}$ model in $1+1$ dimensions in the form

$$
S=\int d^{2} x\left[\frac{1}{2}\left(\partial_{\mu} \phi\right)\left(\partial^{\mu} \phi\right)-\frac{\lambda}{4}\left(\phi_{\star}^{2}-\frac{m^{2}}{\lambda}\right)^{2}\right],
$$

where $\phi_{\star}^{2} \equiv \phi \star \phi$. Star denotes the Moyal product

$$
(f \star g)(x)=\left[\exp \left(\frac{i}{2} \Theta^{\mu \nu} \partial_{\mu}^{x} \partial_{\nu}^{y}\right) f(x) g(y)\right]_{y^{\mu}=x^{\mu}},
$$

where $\Theta^{\mu \nu}$ is a constant skew-symmetric matrix which can be chosen as $\Theta^{\mu \nu}=$ $2 \theta \epsilon^{\mu \nu}$ with $\epsilon^{01}=1$. After splitting the coordinates into time and space, $\left\{x^{\mu}\right\}=$ $\{t, x\}$, we have the following useful formulae

$$
f(x) \star e^{i \omega t}=e^{i \omega t} f(x+\theta \omega), \quad e^{i \omega t} \star f(x)=e^{i \omega t} f(x-\theta \omega)
$$


Obviously, static solutions of the commutative $\phi^{4}$ remain also solutions in the $\mathrm{NC}$ case. In particular, we have the kink solution

$$
\Phi(x)=\frac{m}{\sqrt{\lambda}} \tanh \left(\frac{m x}{\sqrt{2}}\right) .
$$

Classical energy of this soliton is

$$
E_{\mathrm{cl}}=\frac{2 \sqrt{2}}{3} \frac{m^{3}}{\lambda} .
$$

Qualitatively the spectrum of fluctuations above the kink solution in NC $\phi^{4}$ theory was studied in [19]. Here we repeat some steps from that paper. Let us split $\phi=\Phi+\varphi$ with $\Phi$ being the kink solution (4) and $\varphi$ describing quantum fluctuations. The linearized equation of motion reads

$$
-\partial_{t}^{2} \varphi+\partial_{x}^{2} \varphi+m^{2} \varphi-\lambda(\Phi \star \Phi \star \varphi+\Phi \star \varphi \star \Phi+\varphi \star \Phi \star \Phi)=0 .
$$

Since the kink (44) is static, we can look for the solutions of (6) in the form $\varphi=e^{i \omega t} \eta(x)$. By substituting this ansatz in (6) and using the relations (3) we obtain the following equation for $\eta$

$$
\omega^{2} \eta+\partial_{x}^{2} \eta+m^{2} \eta-\lambda\left(\Phi_{+}^{2}+\Phi_{+} \Phi_{-}+\Phi_{-}^{2}\right) \eta=0
$$

where $\Phi_{ \pm}(x)=\Phi\left(x_{ \pm}\right)$and $x_{ \pm}=x \pm \theta \omega$. It is convenient to choose the mass units such that

$$
m=\sqrt{2} .
$$

Then eq. (77) yields

$$
\left(-\partial_{x}^{2}+M^{2}+U(x ; \omega)\right) \eta=\omega^{2} \eta
$$

where

$$
U(x ; \omega)=2\left(\tanh ^{2}\left(x_{+}\right)+\tanh \left(x_{+}\right) \tanh \left(x_{-}\right)+\tanh ^{2}\left(x_{-}\right)-3\right)
$$

and the constant part $M^{2}=4$ is selected in such a way that $U(x ; \omega) \rightarrow 0$ exponentially fast for $x \rightarrow \pm \infty$ and a fixed $\omega$.

\section{Quantum energy of the fluctuations}

The vacuum energy can be formally defined as a sum of eigenfrequencies of quantum fluctuations $E=\frac{\hbar}{2} \sum \omega$. In NC theories with time-space noncommutativity (in particular, in all $\mathrm{NC}$ theories in $(1+1)$ dimensions) it is hard to define a canonical Hamiltonian (due to the presence of an infinite number of time derivatives) and thus to justify this formula for $E$ rigorously. Here we follow [17] and 
accept this definition of the vacuum energy (see the comments made in sec. 1 above). More precisely, after taking $\hbar=1$ we split the vacuum energy as

$$
E=E_{B}+E_{C}
$$

where

$$
E_{B}=\frac{1}{2} \sum \omega_{B}
$$

is a finite sum over the bound state frequencies. The contribution of the continuous spectrum has to be regularized. In the zeta function regularization it reads

$$
E_{C}=\int_{M}^{\infty} d \omega \omega^{1-2 s} \rho(\omega)
$$

where a regularization parameter $s$ has been introduced which should be put zero at the end of the calculations. The function $\rho(\omega)$ is the spectral density in the continuous spectrum. By using the well known relation between the spectral density and the phase shift we can write

$$
E_{C}=\frac{1}{2 \pi} \int_{0}^{\infty} d k\left(k^{2}+M^{2}\right)^{\frac{1}{2}-s} \partial_{k} \delta(k)
$$

where $\omega=\sqrt{k^{2}+M^{2}}$. (For the sake of completeness we rederive this formula for noncommutative case in Appendix A). Equivalently,

$$
E_{C}=\frac{1}{2 \pi} \int_{M}^{\infty} d \omega \omega^{1-2 s} \partial_{\omega} \delta(\omega) .
$$

This quantity is divergent in the limit $s \rightarrow 0$. To get rid of the divergences one has to know the the asymptotic behavior of the spectral density for large frequencies.

\subsection{Asymptotic behavior of the spectral density}

Since the potential in (9) depends on the frequency $\omega$ we are dealing with a non-linear spectral problem. To analyze the spectral density we use a method developed initially in [20-22] and then adapted to NC theories in [18].

Let us consider an auxiliary eigenvalue problem

$$
L(\sigma) \psi_{\sigma, \omega}=\omega^{2} \psi_{\sigma, \omega}
$$

where

$$
L(\sigma)=-\partial_{x}^{2}+M^{2}+U(x ; \sigma)
$$

The functions $\eta_{\omega}=\psi_{\omega, \omega}$ solve our initial eigenvalue problem (9), but the spectral densities are different. Let us denote the density of the auxiliary problem by $\rho(\sigma, \omega)$. Note, that according to Appendix A we are actually working with the 
densities from which (an infinite) contribution from free massive fields in an infinite space has been subtracted. By using this density, one can calculate spectral functions of the operators $L(\sigma)$ (with a fixed $\sigma$ ). In particular, the heat kernel for $L(\sigma)$ reads

$$
K(L(\sigma) ; t)=\operatorname{Tr}\left(e^{-t L(\sigma)}-e^{-t\left(-\partial_{x}^{2}+M^{2}\right)}\right)=\int d \omega e^{-t \omega^{2}} \rho(\sigma, \omega) .
$$

Here Tr denotes a trace over the space of square integrable functions on the real line. Again, a subtraction of the "free" heat kernel is necessary. Note, that the integration in (18) must be extended over the whole spectrum, including the bound states.

In turn, the spectral density can be expressed through the heat kernel by means of an inverse Laplace transformation.

For any fixed real $\sigma$ the operator $L(\sigma)$ is a Laplace type operator with a smooth potential. Therefore, the following asymptotic expansion 1 is valid as $t \rightarrow+0$

$$
K(L(\sigma) ; t) \simeq \sum_{n=1}^{\infty} t^{n-1 / 2} a_{2 n}(\sigma) .
$$

Odd-numbered coefficients vanish since there is no boundary. The coefficient $a_{0}$ does not contribute because of the subtraction of a free heat kernel in (18). Two leading coefficients read

$$
\begin{aligned}
& a_{2}(\sigma)=-(4 \pi)^{-1 / 2} \int d x U(x ; \sigma), \\
& a_{4}(\sigma)=(4 \pi)^{-1 / 2} \int d x\left[\frac{1}{2} U(x ; \sigma)^{2}+M^{2} U(x ; \sigma)\right] .
\end{aligned}
$$

In our case, by using (10) we obtain

$$
a_{2}(\sigma)=\frac{4}{\sqrt{\pi}}(\theta \sigma \operatorname{coth}(2 \theta \sigma)+1) .
$$

For large $\sigma$ we have

$$
a_{2}(\sigma)=\sigma a_{2,1}+a_{2,0}+\text { e.s.t. }
$$

where

$$
a_{2,1}=\frac{4 \theta}{\sqrt{\pi}}, \quad a_{2,0}=\frac{4}{\sqrt{\pi}},
$$

and the corrections in eq. (23) are exponentially small. For the future use we note

$$
a_{2,0}=-\frac{\lambda}{\sqrt{\pi}} \int d x\left(\Phi^{2}-\frac{m^{2}}{\lambda}\right) .
$$

\footnotetext{
${ }^{1}$ For recent reviews on the heat kernel expansion the reader can consult [23] in the commutative case, and [24] on NC spaces.
} 
Here we used explicit form of the kink solution (44) and restored the $m$-dependence on dimensional grounds.

Higher heat kernel coefficients $a_{2 p}$ are integrals of local polynomials constructed from the potential $U(x ; \sigma)$ and its' derivatives. One can easily prove that for large $\sigma$

$$
a_{2 p}(\sigma)=\sigma a_{2 p, 1}+a_{2 p, 0}+\mathcal{O}(1 / \sigma) .
$$

Probably, the corrections above are even exponentially small, but we shall not rely on this.

The spectral density $\rho(\sigma, \omega)$ taken at coinciding arguments is not the density $\rho(\omega)$ of our initial spectral problem (9). As demonstrated in [20-22] (see also [18] for a discussion in the framework of $\mathrm{NC}$ theories) one has to construct another density $\varrho(\sigma, \omega)$ which is related to a heat-kernel like object

$$
\tilde{K}(\sigma ; t)=\left(1+\frac{1}{2 \sigma t} \frac{\partial}{\partial \sigma}\right) K(L(\sigma) ; t)
$$

through the equation

$$
\tilde{K}(\sigma ; t)=\int d \omega \varrho(\sigma, \omega) e^{-t \omega^{2}}
$$

Then

$$
\rho(\omega)=\varrho(\omega, \omega) .
$$

We do not know any differential or pseudo-differential operator $\tilde{L}(\sigma)$ such that $\tilde{K}(\sigma ; t)=\operatorname{Tr}\left(e^{-t \tilde{L}(\sigma)}\right)$. In any case, such $\tilde{L}$ cannot be a Laplacian on the real line with a smooth potential. However, both $\tilde{K}$ and $\varrho$ are well defined, which allows us to consider other spectral functions.

For $t \rightarrow+0$

$$
\begin{aligned}
& \tilde{K}(\sigma ; t) \simeq \sum_{n=0}^{\infty} \tilde{a}_{2 n}(\sigma) t^{-\frac{1}{2}+n} \\
& \tilde{a}_{2 n}(\sigma)=a_{2 n}(\sigma)+\frac{1}{2 \sigma} a_{2 n+2}(\sigma) .
\end{aligned}
$$

For large $\sigma$ we have

$$
\tilde{K}(\sigma ; t) \simeq t^{-1 / 2} \frac{1}{2 \sigma} a_{2,1}+t^{1 / 2}\left(\sigma a_{2,1}+a_{2,0}+\frac{1}{2 \sigma} a_{4,1}\right)+\ldots
$$

We are going to use this expansion to evaluate the large $\omega$ behavior of $\varrho(\sigma, \omega)$, and, after setting $\sigma=\omega$ - the large $\omega$ behavior of the physical density $\rho(\omega)$. As noted in $[22,25]$, the problem is that besides from powers of $\omega$ the high frequency asymptotics of the spectral density contain oscillating terms which are not defined by the heat trace asymptotics. Strictly speaking, the heat kernel expansion defines the asymptotic behavior of the so-called Riesz means of the 
spectral density rather than that of the spectral density itself. This is not precisely what we need, but we learn an important lesson: the power-law asymptotics are defined by the heat kernel expansion. Oscillating terms are less important anyway since corresponding contributions to the vacuum energy are better convergent than that from pure powers.

Let us consider the zeta function corresponding to $\varrho(\sigma, \omega)$

$$
\tilde{\zeta}(\nu)=\int d \omega\left(\omega^{2}\right)^{-\nu} \varrho(\sigma, \omega)
$$

One should be careful with possible contribution from $\omega=0$. One should either treat such state separately, or add a small positive part to the mass. The details are not essential for us since we are interested in the behavior at large $\omega$.

There is a well know relation between residues of the zeta function and the heat kernel coefficients

$$
\tilde{a}_{2 k}=\operatorname{Res}_{\nu=\frac{1}{2}-k} \Gamma(\nu) \tilde{\zeta}(\nu)=\Gamma\left(\frac{1}{2}-k\right) \operatorname{Res}_{\nu=\frac{1}{2}-k} \tilde{\zeta}(\nu) .
$$

On the other hand, if the spectral density has a contribution behaving like $c_{p} \omega^{p}$ at large $\omega$, the zeta function has a pole term

$$
\sim 2 \int_{\Omega}^{\infty} c_{p} \omega^{p-2 \nu} \sim \frac{c_{p}}{\nu-(p+1) / 2},
$$

where $\Omega$ is a large number, and the coefficient of 2 appeared because we have to take into account degeneracy of the continuous spectrum.

Oscillatory terms do not contribute to the poles. Indeed, after analytical continuation from large positive $\nu$ the integral $\int_{\Omega}^{\infty} d \omega \omega^{-\nu} \sin (b \omega)$ has no poles on the real line.

Next we compare (34) with (33) and (30), (31) to obtain the following power law asymptotics of the spectral density

$$
\varrho(\sigma, \omega) \simeq \frac{1}{2 \sqrt{\pi} \sigma} a_{2,1}-\frac{1}{2 \sqrt{\pi}} \omega^{-2}\left(a_{2,1} \sigma+a_{2,0}+a_{4,1} /(2 \sigma)\right)+\ldots
$$

For the physical spectral density we have

$$
\rho(\omega)=\varrho(\omega, \omega) \simeq-\frac{1}{2 \sqrt{\pi}} \omega^{-2} a_{2,0}+\mathcal{O}\left(\omega^{-3}\right)
$$

Note, that all terms with $\omega^{-1}$ cancel against each other 2 . This formula does not contain possible oscillating term which cannot be obtained by these methods.

One should not be afraid of negative spectral densities. We have subtracted the spectral density of a free massive field. What remains can have both signs.

\footnotetext{
${ }^{2}$ The terms with $1 / \omega$ in the spectral density lead to linear divergences in the vacuum energy. In the zeta-function regularization such divergences are removed automatically, but they may cause problems in other regularization schemes.
} 


\subsection{Renormalization}

Let us now consider renormalization of the vacuum energy. Since only the contribution from continuous spectrum is divergent, we treat this term only

$$
E_{C}=\mu^{2 s} \int_{M}^{\infty} d \omega \omega^{1-2 s} \rho(\omega)
$$

where we introduced a parameter $\mu$ with the dimension of mass in order to keep proper dimension of the vacuum energy $E_{C}$ independently of the regularization parameter $s$. Next we choose some frequency $\Omega$ and split the integral into two parts. The part from $M$ to $\Omega$ we leave as it is. In the part from $\Omega$ to infinity we add and subtract the asymptotics $\rho_{\text {as }}(\omega)$ of the spectral density.

$$
\begin{aligned}
E_{C} & =\mu^{2 s} \int_{M}^{\Omega} d \omega \omega^{1-2 s} \rho(\omega)+\mu^{2 s} \int_{\Omega}^{\infty} d \omega \omega^{1-2 s}\left(\rho(\omega)-\rho_{\mathrm{as}}(\omega)\right) \\
& +\mu^{2 s} \int_{\Omega}^{\infty} d \omega \omega^{1-2 s} \rho_{\mathrm{as}}(\omega) .
\end{aligned}
$$

The choice of $\Omega$ is simply a matter of convenience. All ultraviolet divergences are contained in the last term. As $\rho_{\text {as }}$ we take the first term in (36),

$$
\rho_{\mathrm{as}}(\omega)=-\frac{1}{2 \sqrt{\pi}} \omega^{-2} a_{2,0}=-\frac{2}{\pi} \omega^{-2},
$$

where we took into account (24). We assumed that oscillating terms in the spectral densities (which are beyond our control) do not contribute to the divergences. This assumption cannot be universally true. However, in the case we consider in this paper the subtraction of (39) indeed gives a convergent integral (see below).

Therefore, the assumption we made is correct as well as the renormalization of the vacuum energy which will be done in a moment.

The last term in (38) can be easily evaluated,

$$
E_{\mathrm{div}}=\mu^{2 s} \int_{\Omega}^{\infty} d \omega \omega^{1-2 s} \rho_{\mathrm{as}}(\omega)=-\frac{1}{2 \sqrt{\pi}} a_{2,0} \frac{1}{2 s}\left(\frac{\mu}{\Omega}\right)^{2 s}
$$

Near $s=0 E_{\text {div }}$ behaves as

$$
E_{\text {div }}=-\frac{1}{2 \sqrt{\pi}} a_{2,0}\left[\frac{1}{2 s}+\ln \left(\frac{\mu}{\Omega}\right)+\mathcal{O}(s)\right]=-\frac{2}{\pi}\left[\frac{1}{2 s}+\ln \left(\frac{\mu}{\Omega}\right)+\mathcal{O}(s)\right] .
$$

The pole term

$$
E_{\text {pole }}=-\frac{1}{\pi s} \frac{m}{\sqrt{2}}
$$

(where we restored the $m$-dependence by using dimensional considerations) can be removed by an infinite renormalization of the mass

$$
\delta m^{2}=\frac{\lambda}{2 s \pi}
$$


c.f. (5) 3 . This counterterm does not depend on $\theta$ but is $2 / 3$ of the corresponding counterterm in the commutative case $[10,12]$.

After removing the pole one can lift the regularization taking the limit $s \rightarrow 0$.

$$
\begin{aligned}
E= & \frac{1}{2} \sum \omega_{B}+\int_{M}^{\Omega} d \omega \omega \rho(\omega) \\
& +\int_{\Omega}^{\infty} d \omega \omega\left(\rho(\omega)-\rho_{\mathrm{as}}(\omega)\right)-\frac{2}{\pi} \ln \left(\frac{\mu}{\Omega}\right)
\end{aligned}
$$

We remind that $\rho(\omega)=(2 \pi)^{-1} \partial_{\omega} \delta(\omega)$.

The presence of a free parameter $\mu$ reflects the possibility of a finite renormalization. To fix $\mu$ one needs a normalization condition. In the commutative case in $(1+1)$ dimensions it is usually required that the tadpoles are cancelled by counterterms (the "no-tadpole" condition). This condition is formulated on a constant topologically trivial background. In noncommutative theories oneloop divergences on a constant background coincide with the divergences in the commutative case and thus differ from the divergences in the kink sector. (The difference is precisely the $2 / 3$ factor discussed above). Therefore, the no-tadpole condition is not suitable for us. In the case of commutative kink, there is the large mass subtraction scheme [29] which is equivalent to the no-tadpole condition [12]. Although this scheme can be applied even in non-renormalizable theories, it is not clear how to implement it in the noncommutative case. This situation is not hopeless, but it is more natural to address it together with studying the commutative limit $\theta \rightarrow 0$. Our numerical scheme (see below) does not work well in this limit. Therefore, we postpone the discussion of physically motivated normalization conditions until a future publication. Here, for the sake of simplicity, we put

$$
\mu=M
$$

The values of $E$ for other choices of $\mu$ differ by a shift $-(2 / \pi) \ln (\mu / M)$.

\section{Calculation of energy}

Let us start from the first term in the formula (44) which contains the summation over the bound state frequencies. Here we shall distinguish the two cases: first,

\footnotetext{
${ }^{3}$ In principle one can do renormalization directly in the action by using (25). The heat kernel expansion for $\mathrm{NC} \phi^{4}$ constructed in [26] predicts the same multiplier of $2 / 3$ which relates counterterms in commutative and non-commutative cases. On should however keep in mind that the results of [26] are valid for background fields which decay rapidly at the infinity. This is not the case of the kink solution, which tends to different constants at two infinities. Sensitivity of the heat kernel expansion to the asymptotic behavior of background fields is a generic feature of NC manifolds. The only exception are the expansions for operators containing only left or only right Moyal multiplications. In this latter case universal formulae exist as long as corresponding integrals are convergent [27,28].
} 


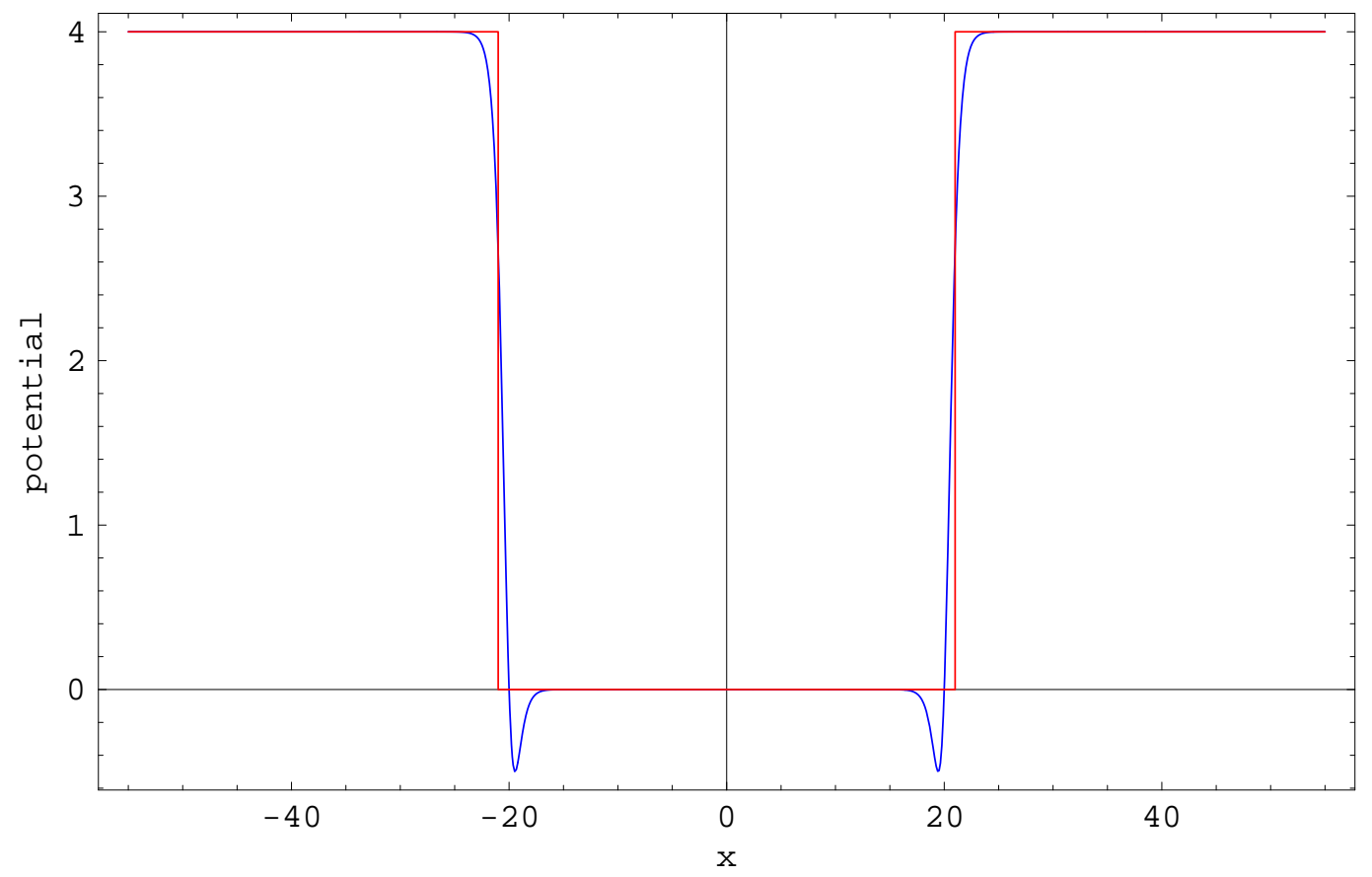

Figure 1: The effective potential $U(x, \omega)+M^{2}$ for $\omega=2, \theta=10$ and its square well approximation.

for $\theta \omega$ considerably larger than 1 , and second, $\theta \omega$ comparable or smaller than 1. When $\theta \omega \gg 1$ we have very good approximation by a square well potential (cf. Fig. (4). The square well potential is, $V=0$ for $|x|>l$ and $V=-V_{0}<0$ for $|x|<l$. (In our case $V_{0}=4$ ). The scattering data for this potential read

$$
\begin{aligned}
& s_{21}=s_{12}=\frac{V_{0} e^{-2 i k l}\left(e^{2 i \omega l}-e^{-2 i \omega l}\right)}{(k+\omega)^{2} e^{-2 i \omega l}-(k-\omega)^{2} e^{2 i \omega l}} \\
& s_{11}=s_{22}=\frac{4 \omega k e^{-2 i k l}}{(k+\omega)^{2} e^{-2 i \omega l}-(k-\omega)^{2} e^{2 i \omega l}}
\end{aligned}
$$

with $\omega=\sqrt{k^{2}+V_{0}}$. The width of an approximating square-well potential must be chosen such that it correctly reproduces the leading asymptotics of the phase shift, i.e. through the condition

$$
-2 l(\omega) V_{0}=\int d x U(x ; \omega)=-4(2+2 \theta \omega \operatorname{Coth}(2 \theta \omega)) .
$$

As $2 l(\omega)=\theta \omega+c$, it immediately follows $c=1$, cf. Fig. 4. (Note, that in [19] the value $c=0$ was taken to estimate the number of bound states for large $\theta$. To the leading order in $\theta$ both approximations coincide, but the one chosen here reproduces the scattering data of $U(x ; \omega)$ with a better accuracy.) 
It is well known from quantum mechanics that the bound states of the square well potential obey the equations

$$
\tan \left(\theta \omega^{2}\right)-\frac{\sqrt{4-\omega^{2}}}{\omega}=0, \quad \tan ^{-1}\left(\theta \omega^{2}\right)+\frac{\sqrt{4-\omega^{2}}}{\omega}=0 .
$$

In the Table I. one can see some bound states calculated with the above formulas (48) for $\theta=10$.

Alternatively, for finding the bound states one can use the well-known WKB formula of the first order

$$
\int_{x_{1}}^{x_{2}} \sqrt{-U\left(x, \omega_{n}\right)+\omega_{n}^{2}} d x=\pi\left(n+\frac{1}{2}\right),
$$

where $x_{1}$ and $x_{2}$ are the turning points. It is well-known that WKB approach works well for the low laying bound states, i.e. the smaller $\theta \omega$, the better accuracy of the WKB approximation. At the same time, the larger $\theta \omega$, the better accuracy of the square well approximation. As a result, one can see in the Table I, with an example of $\theta=10$ case, that highly excited bound state frequencies obtained from the square well approximation agree very well with their WKB values in the regime of large $\theta \omega$. Thus the difference between the WKB and SW (square well) data is less than fractions of one percent. Therefore we expect that relative error of our calculations of energy for large $\theta$ should not exceed one percent. Let us also remind that the zero mode $\omega_{0}=0$ is the same as in the commutative case. (This is the translation zero mode). To summarize, for all bound state frequencies $\omega_{n \geq 1}$ we use the WKB approximation, which is known to be accurate for lower eigenstates, and which practically coincides with the frequencies obtained from the square well approximation near the barrier.

On the contrary to the discrete spectrum, when considering integrals over continuous part of the spectrum (second and third terms in (44)), we start from $\theta=2, \omega=2$ and can use the square well approximation. In other words $\Omega=M$ in (44), if we are limited by not small values of $\theta$. For small $\theta$ and small $\omega$ (of the bound states and in the beginning of the continuous part of the spectrum) the the potential does not look like square well, but rather like a modified PöschlTeller potential. The WKB method is certainly justified for that case as well for calculation of bound states, yet is computationally difficult for the continuous spectrum. That is why we did not consider the limit of small $\theta$.

We used the following expression for the phase shift

$$
\delta=\frac{1}{2 i} \ln \left(s_{11}^{2}-s_{21}^{2}\right)
$$

(cf. Appendix A). The dominant asymptotic behavior of $\rho(\omega)_{\text {as }}$ at large $\omega$ is given by (39) above.

Since we put $\Omega=M$ the second term in (44) vanishes. The calculation of the third term in (44) was done by numerical integration with a $\rho$ function given 
by a square well approximation. The numerical integration was performed by Mathematica and the values for different $\theta$ are given in Fig. 1. One can see there that the roughly linear dependence on $\theta$ takes place. The approximating square well potential differs from the exact potential $U(x ; \omega)$ only slightly near the points $x= \pm(\theta \omega+1)$, and the form of this difference practically does not depend on $\theta$. Therefore, especially since we deal with a massive field, we may hope that the total error will remain small and bounded independently of $\theta$, so that our conclusion about the linear growth of the integral over the continuous spectrum will remain true even if a better approximation is used. Besides, as we shall see, the contribution of the continuous spectrum is about $1 / 2$ of the contribution of the bound states, so that any error in the continuous spectrum is less important. Adding the values for the integration over continuous spectrum (Fig. 1) to the sum over the bound state frequencies (see Table I, except for the case $\theta=7$ which is not presented explicitly in order not too overload the Table), we get

$$
\begin{array}{ll}
E=2.76, & (\theta=2) \\
E=2.95, & (\theta=3) \\
E=4.66, & (\theta=5) \\
E=6.49, & (\theta=7) \\
E=9.16, & (\theta=10)
\end{array}
$$

Let us note that as the contribution to the energy from the integral over continuous spectrum is linear with $\theta$ (see Fig. 1) and the contribution to the bound state is linear as well, the final values for $E$ as a function of $\theta$ is linear in $\theta$ (see Fig. 2).

In the previous section we put $\mu=M$. Values of the vacuum energy for a different choice of $\mu$ are obtained from the one given in (51) by a constant shift, $E \rightarrow E-(2 / \pi) \ln (\mu / M)$. We fixed the mass units so that $m=\sqrt{2}$. In arbitrary units $E$ should be multiplied by $m / \sqrt{2}$. 


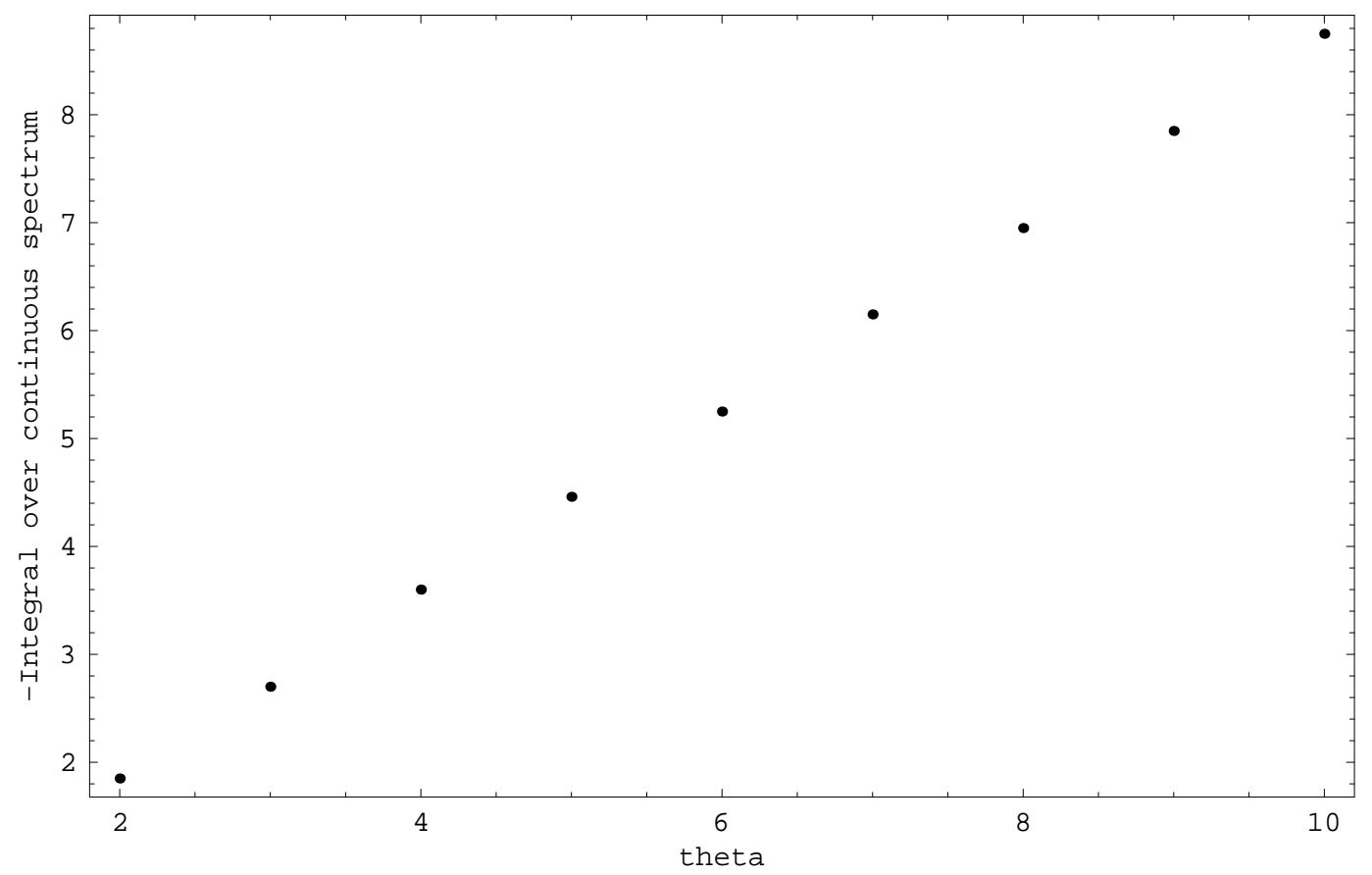

Figure 2: The values of the integral $-\int_{M}^{\infty} d \omega \omega\left(\rho(\omega)-\rho_{\text {as }}(\omega)\right)$ as a function of $\theta$.

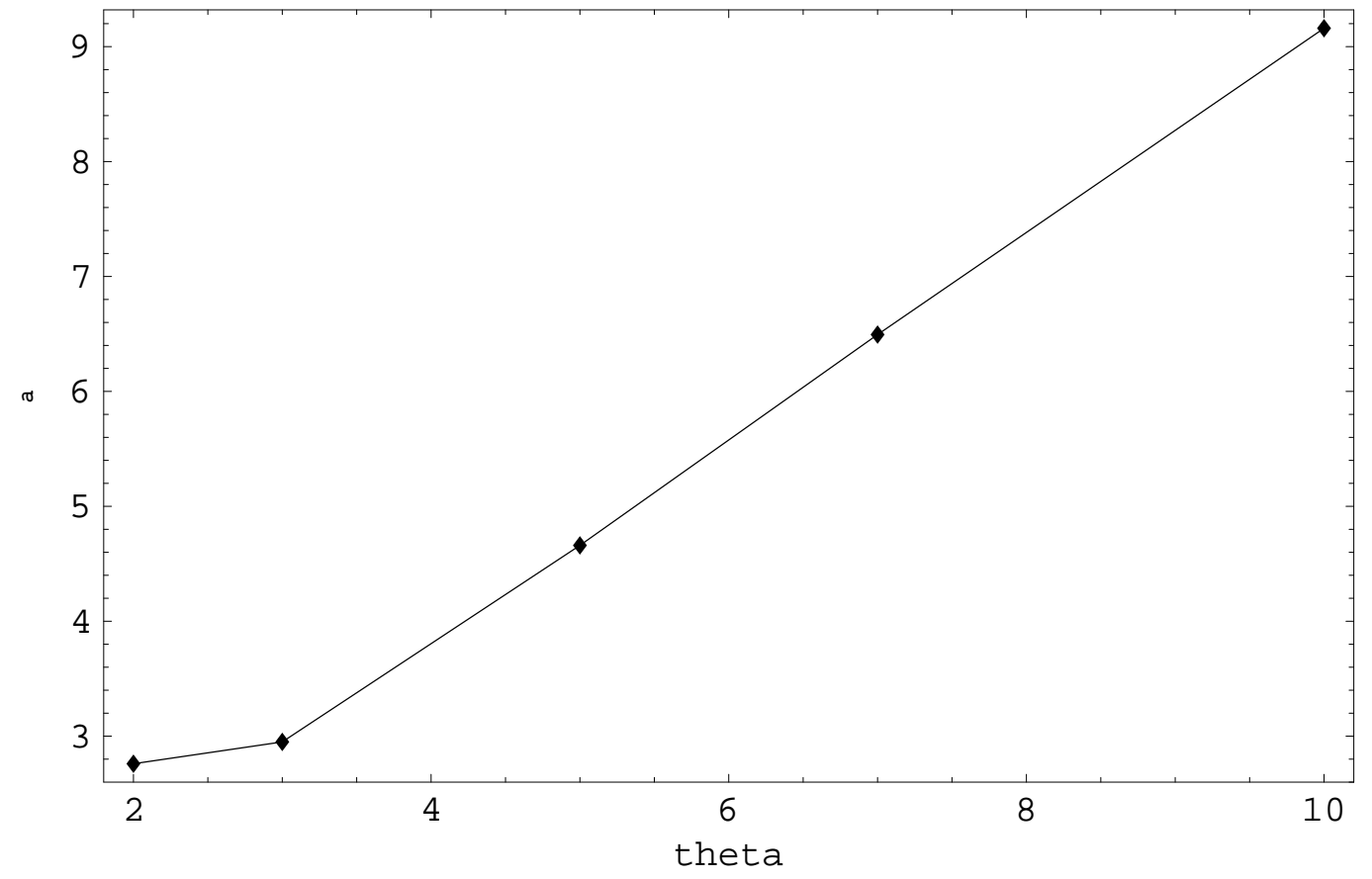

Figure 3: The values of the $E$ as a function of $\theta$. The angle of the slope of the line is: $\arctan \alpha=0.726$. 


\begin{tabular}{|c|c|c|c|}
\hline$\theta=2(W K B)$ & $\theta=3(W K B)$ & $\theta=5(W K B)$ & $\theta=10(W K B)$ \\
\hline$\omega_{1}=0.955$ & $\omega_{1}=0.790$ & $\omega_{1}=0.613$ & $\omega_{1}=0.433$ \\
\hline$\omega_{2}=1.263$ & $\omega_{2}=1.056$ & $\omega_{2}=0.826$ & $\omega_{2}=0.586$ \\
\hline$\omega_{3}=1.496$ & $\omega_{3}=1.260$ & $\omega_{3}=0.995$ & $\omega_{3}=0.708$ \\
\hline$\omega_{4}=1.687$ & $\omega_{4}=1.143$ & $\omega_{4}=1.135$ & $\omega_{4}=0.811$ \\
\hline$\omega_{5}=1.847$ & $\omega_{5}=1.579$ & $\omega_{5}=1.258$ & $\omega_{5}=0.901$ \\
\hline$\omega_{6}=1.970$ & $\omega_{6}=1.712$ & $\omega_{6}=1.368$ & $\omega_{6}=0.983$ \\
\hline- & $\omega_{7}=1.828$ & $\omega_{7}=1.469$ & $\omega_{7}=1.058$ \\
\hline- & $\omega_{8}=1.930$ & $\omega_{8}=1.564$ & $\omega_{8}=1.128$ \\
\hline- & - & $\omega_{9}=1.650$ & $\omega_{9}=1.194$ \\
\hline- & - & $\omega_{10}=1.737$ & $\omega_{10}=1.256$ \\
\hline- & - & $\omega_{11}=1.810$ & $\omega_{11}=1.314$ \\
\hline- & - & $\omega_{12}=1.880$ & $\omega_{12}=1.371$ \\
\hline- & - & $\omega_{13}=1.946$ & $\omega_{13}=1.425$ \\
\hline- & - & - & $\omega_{14}=1.476$ \\
\hline- & - & - & $\omega_{15}=1.526$ \\
\hline- & - & - & $\omega_{16}=1.574$ \\
\hline- & - & - & $\omega_{17}=1.621$ \\
\hline- & - & - & $\omega_{18}=1.665$ \\
\hline- & - & - & $\omega_{19}=1.709$ \\
\hline- & - & - & $\omega_{20}=1.751$ \\
\hline- & - & - & $\omega_{21}=1.793$ \\
\hline- & - & - & $\omega_{22}=1.833$ \\
\hline- & - & - & $\omega_{22 S W}=1.828$ \\
\hline- & - & - & $\omega_{23}=1.872$ \\
\hline- & - & - & $\omega_{23 S W}=1.869$ \\
\hline$\overline{-}$ & - & $\overline{-}$ & $\omega_{24}=1.908$ \\
\hline- & - & - & $\omega_{24 S W}=1.909$ \\
\hline- & - & - & $\omega_{25}=1.944$ \\
\hline- & - & - & $\omega_{25 S W}=1.948$ \\
\hline- & - & - & $\omega_{26}=1.978$ \\
\hline- & - & - & $\omega_{26 S W}=1.985$ \\
\hline
\end{tabular}

Table 1: Values of the bound state frequencies computed by formula (48) (only for the highest five states for $\theta=10$ ) and WKB frequencies found by (49). The comparison shows that the WKB approximation results differ from the SW (square well) approximation only by fractions of one percent. 


\section{Conclusions}

In this work we found that the divergences in the zeta-regularized one-loop vacuum energy of NC kink (defined as a half-sum over egenfrequencies) can be removed by the mass renormalization. This renormalization is, however, different from the one required in the topologically trivial sector. Although the effective potential which defines the spectrum of excitations above the kink depends on frequencies, for intermediate and large values of the $\mathrm{NC}$ parameter $\theta$ the finite part can be calculated by a combination of the WKB method and an approximation by a square well potential. For large noncommutativity the one-loop vacuum energy grows linearly with $\theta$, so that sooner or later it should become larger than the classical value thus signalling break-down of the perturbative expansion.

Our results may be improved and extended in a number of ways. First of all, we need a method of calculations which would work for small $\theta$ and a physically motivated normalization condition to fix the parameter $\mu$. Having these ingredients at hand, one can address the question whether quantum corrections to the NC kink are smooth in the limit $\theta \rightarrow 0$ and whether they reproduce the commutative result [1] in this limit.

\section{A Vacuum energy in the zeta regularization}

Here we derive eq. (14) by using the approach of Bordag [10] and making necessary modifications due to the noncommutativity. In our exposition we also use Ref. [12]. First we introduce a cut-off at large distances by imposing the Dirichlet conditions on the perturbations $\eta(-L(k))=\eta(L(k))=0$. In commutative case, when the potential has no dependence on $\omega$, it is enough to take $L(k)=$ const. In our case the potential does depend on $\omega$. We would like to ensure that $x_{ \pm}$are far away from the boundary for all $\omega$. This can be achieved by taking $L(k)=$ $\theta \omega+L_{0}$ where $L_{0}$ is a large positive constant. Later we shall consider the limit $L_{0} \rightarrow \infty$. The frequency dependent boundary condition is the main novelty here as compared to previous works. We shall demonstrate that it does not change the result.

Without boundaries for each momentum $k$ there are two independent solutions $\eta_{1}, \eta_{2}$ of the wave equation with the asymptotic behavior

$$
\begin{array}{ll}
\eta_{1} \sim e^{i k x}+s_{12} e^{-i k x}, \quad \eta_{2} \sim s_{22} e^{i k x} & \text { for } x \rightarrow-\infty \\
\eta_{1} \sim s_{11} e^{i k x}, \quad \eta_{2} \sim s_{21} e^{i k x}+e^{-i k x} & \text { for } x \rightarrow \infty .
\end{array}
$$

The potential $U$ is symmetric under the reflection $x \rightarrow-x$. Consequently $s_{11}=$ $s_{22}, s_{21}=s_{12}$. For large but finite $L_{0}$ the spectrum is discrete and is defined by the condition

$$
f(k)=\left(\left(s_{11}+s_{21}\right) e^{i k L}+e^{-i k L}\right)\left(\left(s_{11}-s_{21}\right) e^{i k L}-e^{-i k L}\right)=0,
$$


where the bracket with the plus (resp., minus) sign corresponds to a symmetric (resp., antisymmetric) solution.

It is known that if we have discrete spectrum only, the zeta-regularized vacuum energy is a sum over the eigenfrequencies, $E=\frac{1}{2} \sum_{n} \omega_{n}^{1-2 s}=\frac{1}{2} \sum_{n}\left(k_{n}^{2}+M^{2}\right)^{\frac{1}{2}-s}$. The function $\partial_{k} \ln f(k)$ has poles at $k=k_{n}$ with unit residues. Therefore, we can rewrite regularized $E_{C}$ in the form of a contour integral,

$$
E_{C}^{\left(L_{0}\right)}=\frac{1}{2} \oint \frac{d k}{2 \pi i}\left(k^{2}+M^{2}\right)^{\frac{1}{2}-s} \frac{\partial}{\partial k} \ln f(k) .
$$

The integration contour runs anticlockwise around the real positive semiaxis and consists of one branch at $k=\operatorname{Re} k+i \epsilon$, a second branch at $k=\operatorname{Re} k-i \epsilon$, and a small segment $-\epsilon \leq \operatorname{Im} k \leq \epsilon$ along the imaginary axis. Along the upper part of the contour we keep in $f(k)$ only the terms with $\exp (-i k L(k))$ since $\exp (i k L(k))$ vanishes as $L_{0} \rightarrow \infty$. Along the lower part of the contour we retain $\exp (i k L(k))$. The contribution from the third part can be dropped, as in $[10,12]$. We have

$$
E_{C}^{\left(L_{0}\right)}=\frac{1}{2} \int_{0}^{\infty} \frac{d k}{2 \pi i}\left(k^{2}+M^{2}\right)^{\frac{1}{2}-s} \frac{\partial}{\partial k}\left(4 i k L(k)+\ln \left(s_{11}^{2}-s_{22}^{2}\right)\right) .
$$

Next we take into account

$$
s_{11}^{2}-s_{21}^{2}=e^{2 i \delta(k)},
$$

where $\delta(k)$ is the phase shift, and subtract the contribution from free fields of mass $M$ satisfying the same boundary conditions (i.e., the expression (55) with $\delta(k)=0$ ). After taking the limit $L_{0} \rightarrow \infty$ we obtain eq. (14) for the vacuum energy.

Note, that taking the boundaries into account explicitly is essential in supersymmetric theories (where the boundary conditions must be supersymmetric) $[12]$.

\section{Acknowledgments}

R. K. was supported by Japan Society for Promotion of Science (JSPS), Japan. D.V.V. thanks FAPESP for the support.

\section{References}

[1] R. F. Dashen, B. Hasslacher and A. Neveu, "Nonperturbative Methods And Extended Hadron Models In Field Theory. 2. Two-Dimensional Models And Extended Hadrons," Phys. Rev. D 10, 4130 (1974).

[2] R. Rajaraman, Solitons and instantons (Elsivier, Amsterdam, 1996). 
[3] L. D. Faddeev and V. E. Korepin, "Quantum Theory Of Solitons: Preliminary Version," Phys. Rept. 42, 1-87 (1978).

[4] A. Rebhan and P. van Nieuwenhuizen, "No saturation of the quantum Bogomolnyi bound by two-dimensional N = 1 supersymmetric solitons," Nucl. Phys. B 508, 449-467 (1997) arXiv:hep-th/9707163.

[5] H. Nastase, M. A. Stephanov, P. van Nieuwenhuizen and A. Rebhan, "Topological boundary conditions, the BPS bound, and elimination of ambiguities in the quantum mass of solitons," Nucl. Phys. B 542, 471-514 (1999) arXiv:hep-th/9802074.

[6] N. Graham and R. L. Jaffe, "Energy, central charge, and the BPS bound for $1+1$ dimensional supersymmetric solitons," Nucl. Phys. B 544, 432-447 (1999) arXiv:hep-th/9808140.

[7] M. A. Shifman, A. I. Vainshtein and M. B. Voloshin, "Anomaly and quantum corrections to solitons in two-dimensional theories with minimal supersymmetry," Phys. Rev. D 59, 045016 (1999) arXiv:hep-th/9810068.

[8] A. Rebhan, P. van Nieuwenhuizen and R. Wimmer, "New developments in the quantization of supersymmetric solitons (kinks, vortices and monopoles)," Braz. J. Phys. 34, 1273-1287 (2004) arXiv:hep-th/0404223.

[9] M. Shifman and A. Yung, "Supersymmetric Solitons and How They Help Us Understand Non-Abelian Gauge Theories," arXiv:hep-th/0703267.

[10] M. Bordag, "Vacuum energy in smooth background fields," J. Phys. A 28 (1995) 755.

[11] G. V. Dunne, "Derivative expansion and soliton masses," Phys. Lett. B 467, 238-246 (1999) arXiv:hep-th/9907208.

[12] M. Bordag, A. S. Goldhaber, P. van Nieuwenhuizen and D. Vassilevich, "Heat kernels and zeta-function regularization for the mass of the SUSY kink," Phys. Rev. D 66 (2002) 125014 arXiv:hep-th/0203066.

[13] A. Alonso Izquierdo, W. Garcia Fuertes, M. A. Gonzalez Leon and J. Mateos Guilarte, "Generalized zeta functions and one-loop corrections to quantum kink masses," Nucl. Phys. B 635, 525-557 (2002) arXiv:hep-th/0201084.

[14] A. A. Izquierdo, W. G. Fuertes, M. A. G. Leon, M. . d. la Torre Mayado, J. M. Guilarte and J. M. M. Castaneda, "Lectures on the mass of topological solitons," arXiv:hep-th/0611180.

[15] M. R. Douglas and N. A. Nekrasov, "Noncommutative field theory," Rev. Mod. Phys. 73, 977-1029 (2001) arXiv:hep-th/0106048. 
[16] O. Lechtenfeld, "Noncommutative solitons," arXiv:hep-th/0605034.

[17] S. Kurkcuoglu and O. Lechtenfeld, "Quantum Aspects of the Noncommutative Sine-Gordon Model," JHEP 0709, 020 (2007) arXiv:0708.1310 [hepth]].

[18] A. V. Strelchenko and D. V. Vassilevich, "On space-time noncommutative theories at finite temperature," Phys. Rev. D 76, 065014 (2007) arXiv:0705.4294 [hep-th]].

[19] D. V. Vassilevich and A. Yurov, "Space-time non-commutativity tends to create bound states," Phys. Rev. D 69, 105006 (2004) arXiv:hep-th/0311214.

[20] D. V. Fursaev, "Kaluza-Klein method in theory of rotating quantum fields," Nucl. Phys. B 596, 365 (2001) [Erratum-ibid. B 664, 403 (2003)] [arXiv:hep-th/0006217].

[21] D. Fursaev and A. Zelnikov, "Thermodynamics, Euclidean gravity and Kaluza-Klein reduction," Class. Quant. Grav. 18, 3825 (2001) arXiv:hep-th/0104027.

[22] D. V. Fursaev, "Spectral asymptotics of eigen-value problems with non-linear dependence on the spectral parameter," Class. Quant. Grav. 19, 3635 (2002) [Erratum-ibid. 20, 565 (2003)] arXiv:hep-th/0201219].

[23] D. V. Vassilevich, "Heat kernel expansion: User's manual," Phys. Rept. 388, 279 (2003) arXiv:hep-th/0306138.

[24] D. V. Vassilevich, "Heat Trace Asymptotics on Noncommutative Spaces," SIGMA 3 (2007), 093, 11 pages [arXiv:0708.4209 [hep-th]].

[25] S. A. Fulling, "Some properties of Riesz means and spectral expansions", Electron. J. Differ. Eqs. No. 6, 1-39 (1999) arXiv:physics/9710006

[26] D. V. Vassilevich, "Heat kernel, effective action and anomalies in noncommutative theories," JHEP 0508, 085 (2005) arXiv:hep-th/0507123.

[27] D. V. Vassilevich, "Non-commutative heat kernel," Lett. Math. Phys. 67, 185-194 (2004) arXiv:hep-th/0310144.

[28] V. Gayral and B. Iochum, "The spectral action for Moyal planes," J. Math. Phys. 46, 043503 (2005) arXiv:hep-th/0402147].

[29] M. Bordag, K. Kirsten and D. Vassilevich, "On the ground state energy for a penetrable sphere and for a dielectric ball," Phys. Rev. D 59, 085011 (1999) arXiv:hep-th/9811015. 